\title{
Clinical Outcomes of Novel Long-Tapered Sirolimus-Eluting Coronary Stent System in Real-World Patients With Long Diffused De Novo Coronary Lesions
}

\author{
Suresh V. Patted ${ }^{a}$, i, Rajendra Kumar Jain ${ }^{\text {b }}$, P. A. Jiwani ${ }^{\mathrm{b}}$, Satish Suryavanshic, T. R. Raghu ${ }^{\mathrm{d}}$, \\ Hema Raveeshe, S. Rajalakshmif ${ }^{\mathrm{f}}$, Ashok S. Thakkarg, i, Prakash Kumar Turiya ${ }^{\mathrm{g}}$, \\ Priyanka J. Desaig, Anmol Suresh Patted ${ }^{\text {a }}$, Kamal H. Sharma ${ }^{\text {h }}$
}

\begin{abstract}
Background: When coronary lesions involve segments $>48 \mathrm{~mm}$, the only treatment possibility is stent overlapping which is associated with higher neointimal proliferation that lead to more restenosis. Furthermore, tapering of coronary arteries is a major challenge observed with long diffuse coronary lesions. This study attempted to assess the safety and performance of world's first commercialised long-tapered $(60 \mathrm{~mm})$ sirolimus-eluting coronary stent (SES) system for the treatment of long diffused de novo coronary lesions in real world scenario.
\end{abstract}

Methods: This was a retrospective, non-randomised, multicentre study which included 362 consecutive patients implanted with longtapered BioMime ${ }^{\mathrm{TM}}$ Morph SES system for the treatment of long diffused de novo coronary lesions. Safety endpoint was major adverse cardiac events (MACE), which was defined as composite of cardiac death, myocardial infarction (MI) and ischemic-driven target lesion revascularization (ID-TLR), at 12-month follow-up.

Results: Out of 362 patients included, $170(47.0 \%)$ were diabetic and $159(43.9 \%)$ were hypertensive. The mean age of all patients was

Manuscript submitted October 18, 2018, accepted October 25, 2018

${ }^{a}$ KLE Academy of Higher Education and Research Centre (KLE University), Belgaum, Karnataka, 590010, India

bKIMS Hospital, Hyderabad, Telangana, 500003, India

'Fortis Escorts Heart Centre, Raipur, Chhattisgarh, 492001, India

${ }^{\mathrm{d} S}$ Sri Jayadeva Institute of Cardiovascular Sciences and Research, Bengaluru, Karnataka, 560069, India

eSri Jayadeva Institute of Cardiovascular Sciences and Research K.R. Hospital Campus, Mysore, Karnataka, 570016, India

fSUT Hospital Pattom, Trivandrum, Kerala, 695004, India

gMeril Life Sciences Pvt. Ltd., Vapi, Gujarat, 396191, India

hU.N. Mehta Institute of Cardiology, Civil Hospital, Ahmedabad, Gujarat, 380016, India

${ }^{\mathrm{i} C}$ Corresponding Author: Suresh V. Patted, Department of Cardiology, KLE Academy of Higher Education and Research (KLE University), Belgaum, 590010, Karnataka, India. Email: drpatted@yahoo.com; Ashok S. Thakkar, Clinical Research and Medical Writing, Meril Life Sciences Pvt. Ltd., Vapi, Gujarat, 396191, India. Email: ashok.thakkar@merillife.com

doi: https://doi.org/10.14740/cr795
$61.09 \pm 9.04$ years. A total of 625 lesions were identified; out of which 402 lesions were intervened successfully using BioMime Morph. The cumulative incidence of MACE was 7 (2.0\%) at 12-month follow-up which included four $(1.1 \%)$ cardiac deaths, one $(0.3 \%)$ case of MI and two $(0.6 \%)$ ID-TLR. Acute stent thrombosis was reported in one $(0.3 \%)$ patient.

Conclusions: The present study confirms the safety and performance of BioMime Morph, and hence, can be considered as a treatment of choice for long diffused tapered de novo coronary lesions in routine clinical practice.

Keywords: De novo; Long diffused lesions; Major adverse cardiac events; Tapered coronary stent system

\section{Introduction}

Percutaneous coronary intervention (PCI) with stent implantation is the most widely used treatment strategy for coronary artery disease (CAD). Moreover, current generation drug-eluting coronary stents (DES) have remarkable features to improve the clinical outcomes of PCI [1]. However, treatment of long diffused lesions is still a challenge for interventional cardiologists, especially in tapered coronary arteries where the vessel diameter discrepancies may warrant using more than one stent for a long lesion.

Taper is defined as the ratio of the area change to the vessel length. Positive taper refers to a narrowing in cross-section while negative taper refers to an expansion in luminal area [2]. A study by Zubaid et al investigated the magnitude of angiographic tapering in 13 men and 13 women with normal coronary arteries by estimating tapering index (i.e., a difference between the proximal and distal segment diameter, divided by the proximal segment diameter). It was found that proximal and distal left anterior descending artery taper by $18.14 \%$ and $29.9 \%$ respectively, while the proximal and distal right coronary artery taper by $9.12 \%$ and $7.8 \%$ respectively, in both men and women [3].

The strategies adopted for treatment of long diffused lesions in tapered arteries include the use of either multiple stents 


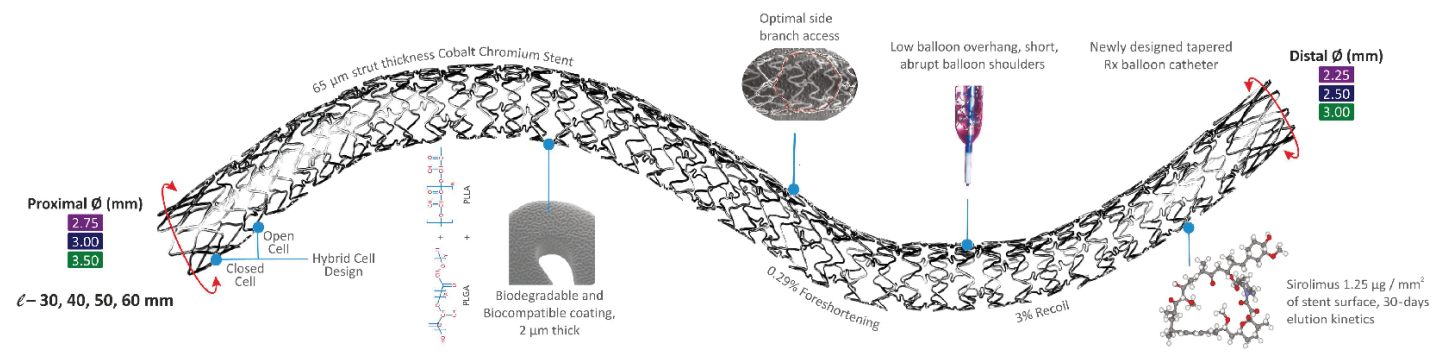

Figure 1. Structure and design of long-tapered BioMime ${ }^{\mathrm{TM}}$ Morph SES system.

or a single long stent, both of which are associated with clinical failure due to the potential risk of mechanical mismatch of stent size $[1,4,5]$. Multiple short stents with variable diameters are often implanted (overlapping) to match the size of the longtapered lesions adequately. However, this solution comes with its own set of limitations, including stent fracture due to vessel rigidity, restenosis because of higher vascular injury, delayed healing, very late stent thrombosis (ST), vessel aneurysm, side branch jailing, increased treatment cost, overuse of anti-restenosis drug and increased exposure to radiation and contrast media [6]. Moreover, implantation of more than one stent might not be necessary or can be completely avoided [7]. Apart from undesirable short-term outcomes, it has been reported that overlapping stents are associated with adverse long-term clinical outcomes, including death or myocardial infarction (MI) [8].

The BioMime ${ }^{\mathrm{TM}}$ Morph (Meril Life Sciences Pvt. Ltd., India), a novel long-tapered biodegradable polymer coated sirolimus-eluting coronary stent (SES) system, has been developed to address the aforementioned issues with conventional stents. A single long-tapered BioMime Morph SES system is often enough for treating long diffused lesion in tapered arteries, and therefore, the local arterial walls can be saved from over-exposure to drug/metal and its related adverse events such as delayed healing, peri-procedural MI, risk of target lesion revascularization and very late ST, which are caused due to use of multiple stents. The purpose of this study was to evaluate the safety and performance of long-tapered BioMime Morph SES system in consecutive real-world patients with long diffused de novo coronary lesions.

\section{Materials and Methods}

\section{Study design and population}

This was a retrospective, non-randomized, multicentre study. The study included a total of 362 consecutive patients (aged $\geq 18$ years), between November 2014 and June 2017 at five clinical centers across India. All the included consecutive patients had undergone the treatment of long diffuse de novo coronary lesion by implantation of at least one long-tapered BioMime Morph SES system. The study was conducted in accordance with the privacy policy of each investigational site, which included their rules and regulations for appropriate use of data in patient-oriented research. This study was in accordance with the Declaration of Helsinki and was approved by the ethics committee. Relevant informed consents were obtained for all the participants prior to the procedure.

\section{Study device and procedure}

The BioMime Morph (Fig. 1) is a novel long-tapered biodegradable polymer coated SES system for the treatment of long diffused coronary lesions. It uses an ultra-thin strut $(65 \mu \mathrm{m})$, cobalt-chromium platform having a unique hybrid design of open and closed cells with uniformly thin coating $(2 \mu \mathrm{m})$ of bioabsorbable polymers viz. PLLA (poly-L-lactic acid) and PLGA (poly-lactic-co-glycolic acid). The stent elutes sirolimus (1.25 $\mu \mathrm{g} / \mathrm{mm}^{2}$ ) within 30 - 40 days after the implantation. Currently available lengths of the BioMime Morph are $30 \mathrm{~mm}, 40 \mathrm{~mm}, 50$ $\mathrm{mm}$ and $60 \mathrm{~mm}$. The stent inflation is guided by the tapered balloon and is estimated to achieve the diameters (proximal to distal) of $2.75-2.25 \mathrm{~mm}, 3.0-2.50 \mathrm{~mm}, 3.50-2.75 \mathrm{~mm}$, and 3.50 - $3.0 \mathrm{~mm}$. The device is "Conformite Europeene" (CE) marked.

PCI was performed according to the standard treatment guidelines followed by each participating center [9]. Pre-dilatation and post-dilatation were carried out as per the operator's discretion. Before the procedure, a loading dose of aspirin (300 $\mathrm{mg}$ ) and of a second anti-platelet agent (clopidogrel, ticagrelor, or prasugrel, according to the clinical settings and operator's preference) were administered in all included consecutive patients. Post-procedure, all patients were recommended for dual antiplatelet therapy for at least 12 months, and aspirin (75 $100 \mathrm{mg}$ once daily) indefinitely beyond the first year. Clinical follow-up was performed at 1 month and the clinical/telephonic follow-up was performed at 6 and 12-month after the index procedure and as when needed based on symptoms.

\section{Endpoints and definitions}

The safety endpoints were the occurrence of major adverse car- 
diac events (MACE) at 6 and 12-month follow-up after the index procedure. MACE was defined as the composite of cardiac death, MI attributed to the target vessel, and ischemia-driven target lesion revascularization (ID-TLR). MI was defined as development of new pathological $\mathrm{Q}$ waves on electrocardiogram, or elevation of creatinine kinase $(\mathrm{CK}) \geq 2$ fold the upper limit of normal with elevated CK-MB in the absence of new pathological Q waves or new ischemic symptoms (e.g., chest pain or shortness of breath) [10]. Cardiac death was defined as any death resulting from an acute MI, sudden cardiac death, death due to heart failure or death due to stroke. ID-TLR was defined as any repeat PCI of the target lesion or coronary artery bypass grafting for the lesion in the previously treated segment or within the $5 \mathrm{~mm}$ proximal or distal to the stent site or edge of DES inflation. ST was classified according to the definitions of the Academic Research Consortium [11]. Procedural success was defined as a successful PCI without in-hospital major clinical complications which included death, MI, and ID-TLR. Device success was defined as the deployment of the study stent at the intended target lesion with the attainment of final residual stenosis of $<30 \%$ of the target lesion estimated by angiography or by visual estimation.

\section{Statistical analysis}

Categorical data were presented as frequency and percentages. Continuous variables were presented as mean \pm standard deviation. All data were analyzed using the Statistical Package for Social Sciences, version 20 (SPSS, Chicago, IL, USA). The survival probabilities were calculated using the Kaplan-Meier method.

\section{Results}

\section{Baseline demographic and clinical characteristics}

The data of 362 consecutive patients who were implanted BioMime Morph, were collected in the study, which included majority of males $(290 ; 80.1 \%)$. Mean age of patients was $61.09 \pm$ 9.04 years. Diabetes mellitus and hypertension were present in $170(47.0 \%)$ and $159(43.9 \%)$ patients, respectively which are conventional risk factors for CAD. Majority of patients (110; $30.4 \%$ ) presented with refractory stable angina. Baseline demographics and clinical characteristics of patients are shown in Table 1.

\section{Lesion and procedural characteristics}

Lesion and procedural characteristics are illustrated in Table 2. A total of 625 lesions were identified in 362 consecutive patients. Out of which, 402 long diffused de novo type C coronary lesions (1.11 lesions per patient) with average lesion length of $40.25 \pm 5.54 \mathrm{~mm}$ were successfully intervened with 390 BioMime Morph novel long-tapered SES system, 144 lesions were treated with 132 other stents, and 79 lesions were not treated with stents (Tables 3, 4). Majority of stents were implanted in the left anterior descending artery $(236 ; 58.7 \%)$ followed by in right coronary artery $(128 ; 31.8 \%)$, left circumflex artery $(33 ; 8.2 \%)$, and left main coronary artery $(5 ; 1.2 \%)$. The average length of the implanted BioMime Morph was 44.85 $\pm 10.23 \mathrm{~mm}$ along with average diameters of $3.2 \pm 0.29 \mathrm{~mm}$ (proximal) and $2.70 \pm 0.29 \mathrm{~mm}$ (distal). Immediate procedural and device success rate was $99.7 \%$ and $100 \%$, respectively.

\section{Clinical outcomes at follow-up}

Clinical follow-up was completed in $98.1 \%$ of included patients at 6 and 12-month (Table 5). Out of 362 patients, seven (1.9\%) were lost to follow-up (Fig. 2). Cumulative incidence of MACE at 6 and 12-month was four $(1.1 \%)$ and seven $(2.0 \%)$, respectively. There were four $(1.1 \%)$ cardiac deaths, one $(0.3 \%) \mathrm{MI}$ and two $(0.6 \%)$ ID-TLRs at 12 -month follow-up. The Kaplan-Meier method and survival rate performed timeto-event analysis of $12-$ month was found to be $98.0 \%$ (Fig. 3). There was a single $(0.3 \%)$ incidence of acute ST immediately after the index procedure and was attributed to resistance to clopidogrel as documented by platelet aggregometry later on. The patient was immediately treated with plain only balloon angioplasty and was stabilized, and discharged 3 days later.

\section{Discussion}

In the current study, long-tapered BioMime Morph SES system has demonstrated safety and performance in consecutive real-world patients with long diffused de novo coronary lesions. In addition to the high $(99.7 \%)$ procedural and $(100 \%)$ device success, the cumulative MACE rate was four $(1.1 \%)$ and seven $(2.0 \%)$ at 6 and 12 -month follow-up, respectively and there was one $(0.3 \%)$ case of acute ST immediately after the procedure.

Studies have shown that the dimensions of coronary arteries taper naturally along with their length. In one study, Banka et al analyzed tapered coronary anatomy, $1 \mathrm{~cm}$ proximal and distal to the stenosis, in 100 consecutive coronary arteries. They observed that $23 \%$ of arteries had $\geq 1 \mathrm{~mm}$ taper and $19 \%$ arteries had a $0.5-0.99 \mathrm{~mm}$ taper [12]. Hence, stent sizing is critical for the success of PCI for treating long-tapered lesions as "stent over-sizing" (stent larger in diameter than the healthy artery) may induce pathological stress on the arterial wall and damage to the artery may be irreversible leading to aneurysm formation, late ST and even late perforations. This may also lead to accelerated vascular injury and augmented neointima formation, leading to in-stent restenosis; whereas "stent under-sizing" (stent smaller in diameter than the healthy artery) may lead to ST as a consequence of stent malposition [13]. In light of these, tapered stents have been developed to minimize clinical failure and maximize the clinical benefits in such patients. The long-tapered BioMime Morph SES system comes with a tapered balloon and is designed to undergo "morphology-mediated" expansion and therefore prevents artery dissection or over-inflation at the distal end of the le- 
Table 1. Baseline Demographics and Clinical Characteristics

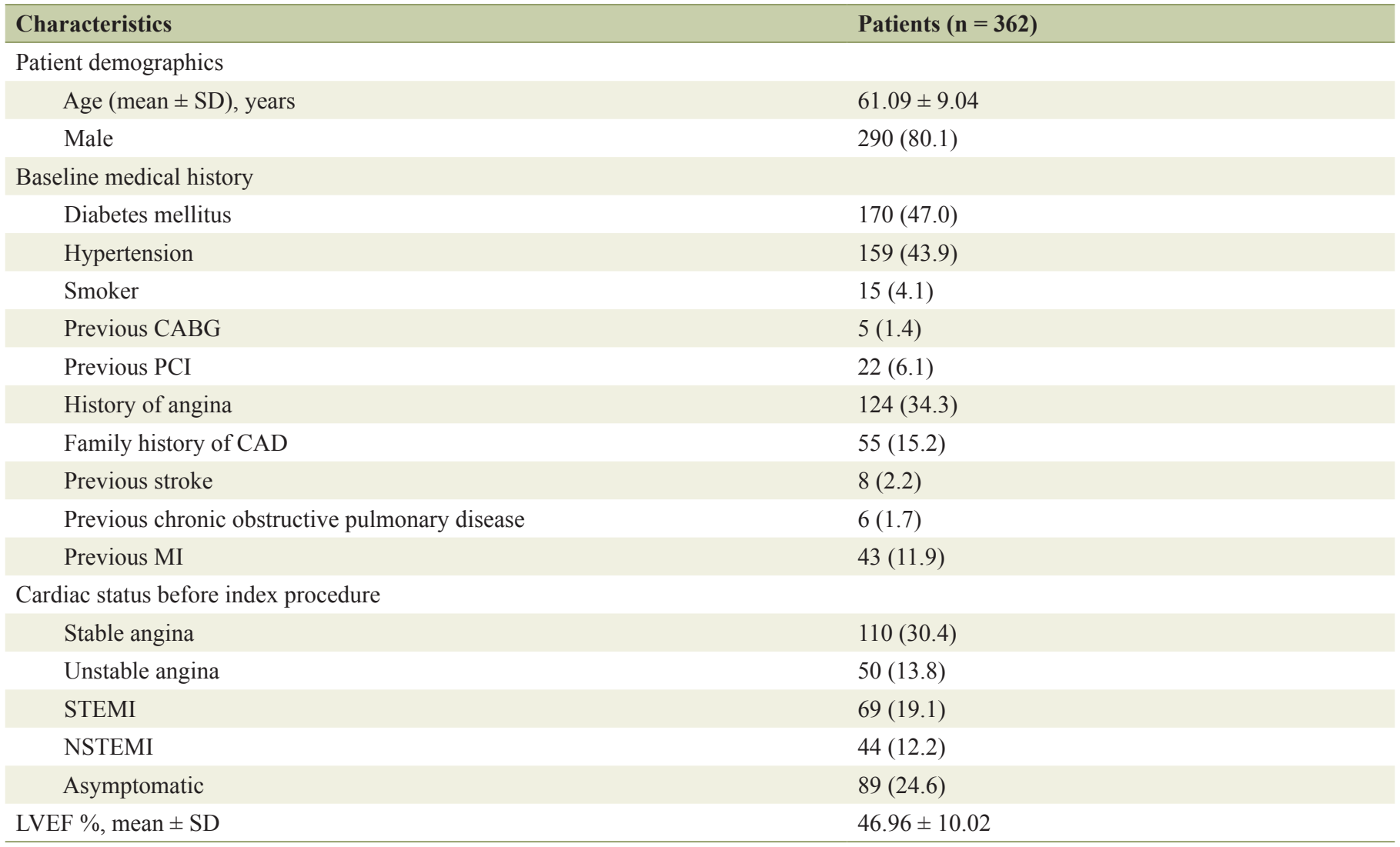

CABG: coronary artery bypass grafting; CAD: coronary artery disease; LVEF: left ventricular ejection fraction; MI: myocardial infarction; NSTEMI: non ST-elevation myocardial infarction; PCI: percutaneous coronary intervention; STEMI: ST-elevation myocardial infarction. Values are presented as frequency (percentage).

\section{Table 2. Lesion and Procedural Characteristics}

\begin{tabular}{ll}
\hline Characteristics & Patients $(\mathbf{n}=\mathbf{3 6 2}) / \mathbf{l e s i o n s}(\mathbf{n}=\mathbf{6 2 5})$ \\
\hline Total number of lesions treated with BioMime Morph SES system & 402 \\
Total number of lesions treated with other stents & 144 \\
Unknown/ no stent treated lesions & 79 \\
\hline Target lesion location & $236 / 402(58.7)$ \\
$\quad$ Left anterior descending artery & $128 / 402(31.8)$ \\
$\quad$ Right coronary artery & $33 / 402(8.2)$ \\
$\quad$ Left circumflex artery & $5 / 402(1.2)$ \\
$\quad$ Left main & $1.11(402 / 362)$ \\
$\quad$ Lesion per patient & \\
Disease vessel & $156 / 362(43.1)$ \\
$\quad$ Single vessel disease & $125 / 362(34.5)$ \\
$\quad$ Double vessel disease & $81 / 362(22.4)$ \\
$\quad$ Triple vessel disease & $40.25 \pm 5.54$ \\
\hline Average target lesion length (mm), Mean \pm SD & $86.10 \pm 9.50$ \\
\hline Occlusion, Mean \pm SD &
\end{tabular}

Values are presented as frequency (percentage). 
Table 3. BioMime Morph SES system characteristics

\begin{tabular}{|ll|}
\hline Characteristics & Patients $(\mathbf{n}=\mathbf{3 6 2})$ \\
\hline Stent length $(\mathrm{mm})$ & \\
\hline 30 & $77 / 390(19.74)$ \\
40 & $125 / 390(32.05)$ \\
50 & $110 / 390(28.21)$ \\
\hline 60 & $78 / 390(20.00)$ \\
Average stent length $(\mathrm{mm})$, mean \pm SD & $44.85 \pm 10.23$ \\
Stent diameter $(\mathrm{mm})$ & \\
\hline $2.75-2.25$ & $53 / 390(13.59)$ \\
$3.00-2.50$ & $151 / 390(38.72)$ \\
$3.5-2.75$ & $15 / 390(3.85)$ \\
\hline $3.5-3.0$ & $171 / 390(43.85)$ \\
Average proximal stent diameter $(\mathrm{mm})$, mean \pm SD & $3.2 \pm 0.29$ \\
\hline Average distal stent diameter $(\mathrm{mm})$, mean \pm SD & $2.70 \pm 0.29$ \\
BioMime Morph SES system per patient & $1.08(390 / 362)$ \\
\hline
\end{tabular}

Values are presented as frequency (percentage).

sion. In fact, few case studies have reported that using longtapered stent offers the advantage of favorable adaptation to vessel tapering, vessel size, and good apposition in patients with long-tapered coronary lesions along with good coronary flow $[14,15]$.

Table 4. Other Implanted Stent Characteristics

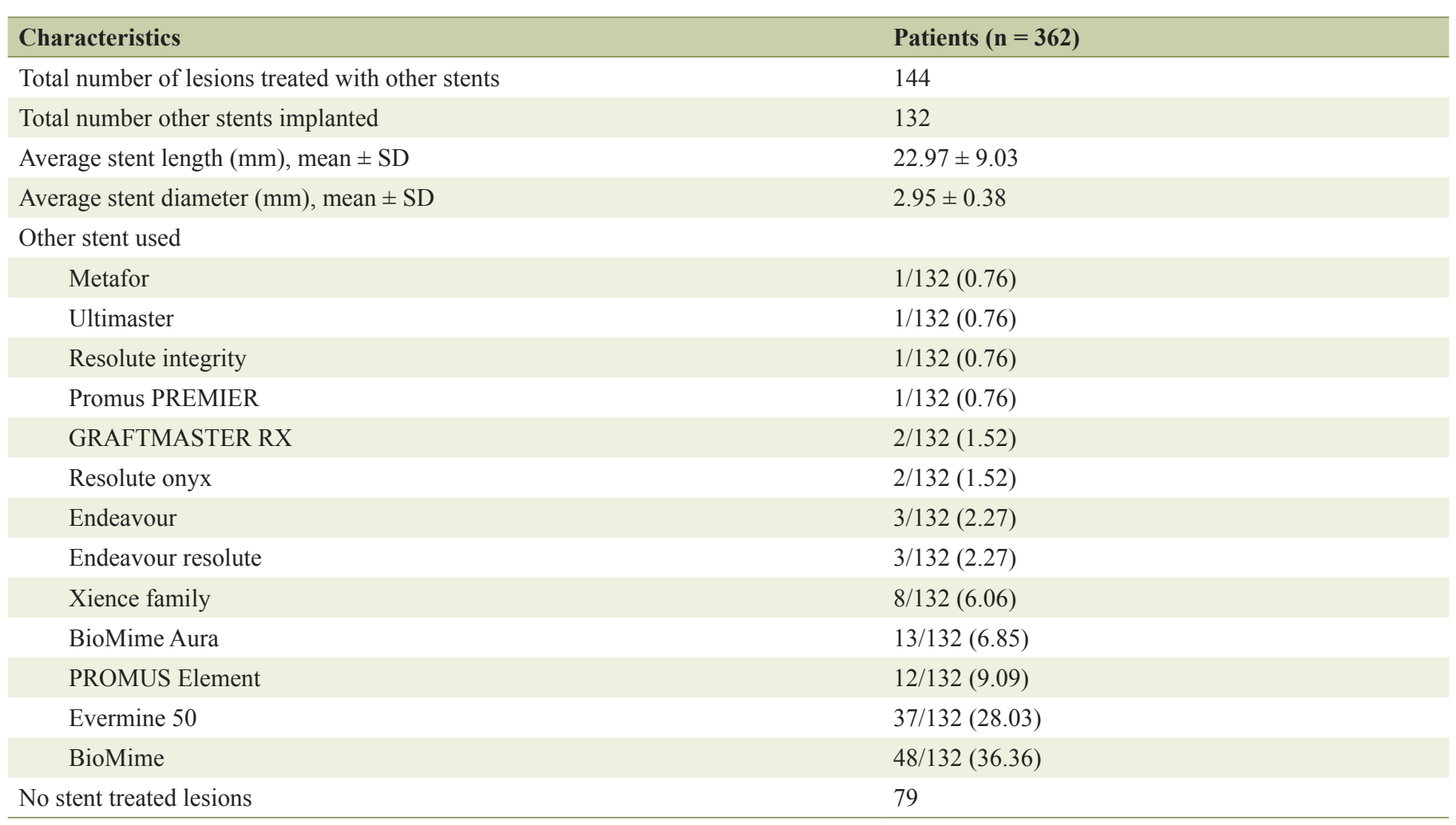

Values are presented as frequency (percentage).

Valero et al assessed safety and efficacy of the BioMime Morph in prospective clinical cohort study which included 50 consecutive patients in whom the implantation of a $60 \mathrm{~mm}$ long DES was attempted due to the presence of coronary lesions longer than $48 \mathrm{~mm}$ (78\% coronary lesions longer than 48 
Table 5. Cumulative Major Adverse Events at 1, 6 and 12-Month Follow-Up

\begin{tabular}{|c|c|c|c|c|}
\hline \multirow{2}{*}{ Events } & In-hospital, n (\%) & 1-month, n (\%) & 6-month, n (\%) & 12-month, n (\%) \\
\hline & $n=362(100 \%)$ & $\mathrm{n}=362(100 \%)$ & $\mathrm{n}=355(98.1 \%)$ & $n=355(98.1 \%)$ \\
\hline All cause death & $0(0.0)$ & $2(0.6)$ & $4(1.1)$ & $6(1.7)$ \\
\hline Non-cardiac death & $0(0.0)$ & $1(0.3)$ & $2(0.6)$ & $2(0.6)$ \\
\hline MI & $0(0.0)$ & $1(0.3)$ & $1(0.3)$ & $1(0.3)$ \\
\hline Acute & $1(0.3)$ & $1(0.3)$ & $1(0.3)$ & $1(0.3)$ \\
\hline Sub-acute & $0(0.0)$ & $0(0.0)$ & $0(0.0)$ & $0(0.0)$ \\
\hline Late & $0(0.0)$ & $0(0.0)$ & $0(0.0)$ & $0(0.0)$ \\
\hline MACE & $0(0.0)$ & $2(0.6)$ & $4(1.1)$ & $7(2.0)$ \\
\hline
\end{tabular}

n: number of patients; MI: myocardial infarction; ID-TLR: ischemia-driven target lesion revascularization; ID-TVR: ischemia driven target vessel revascularization; MACE: major adverse cardiac events; ST: stent thrombosis. Values are presented as frequency (percentage).

$\mathrm{mm}$ and $22 \%$ multiple severe tandem lesions involving arterial segments longer than $48 \mathrm{~mm}$ ) on angiography. The authors reported optimal angiographic result in $46(92 \%)$ patients despite unfavorable anatomy. There was no angiographic distal vessel dissection which can be explained by the tapered design of the stent. Moreover, all the procedures were performed with an acceptable mean fluoroscopy time $(17 \pm 9 \mathrm{~min})$ and mean contrast volume (168 $\pm 49 \mathrm{~mL}$ per procedure) when compared to other series reported in the treatment of long coronary lesions [16]. Zivelonghi et al demonstrated safety and feasibility of the long-tapered BioMime Morph SES system for treatment of the long lesion with chronic total occlusion. The authors noted a high device success rate (only one failure) with the low incidence of peri-procedural complications (one dissection; one perforation leading to pericardial hematoma and one periprocedural MI) and promising short-term clinical outcomes for

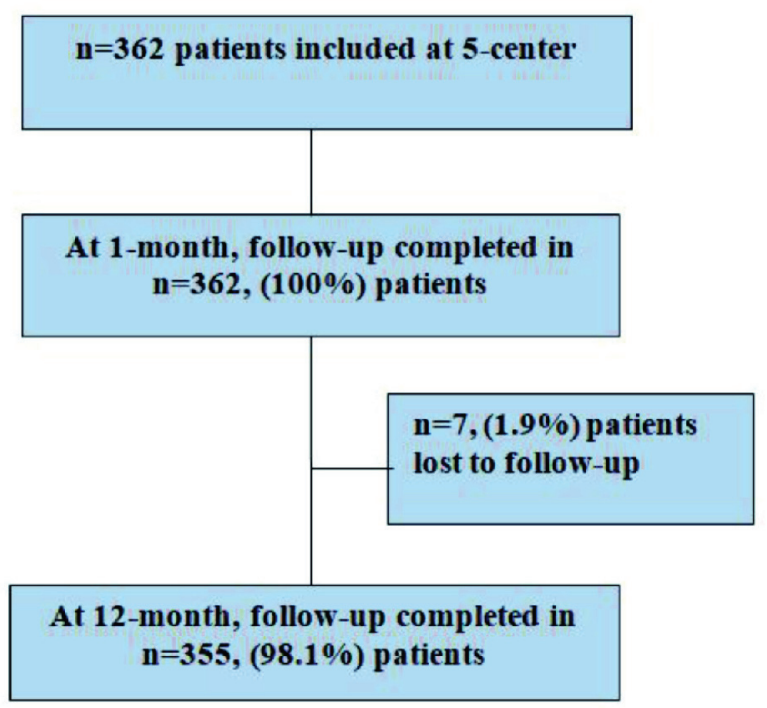

Figure 2. Patients follow-up details at 1 and 12-month.
BioMime Morph at a mean follow-up of 10 months [17]. In addition, satisfactory clinical outcomes (9.4\% MACE with no ST at 12-month clinical follow-up) with the use of very longtapered BioMime stent $(60 \mathrm{~mm})$ were reported by Matchin et al [18]. Also, Qiao Shu Bin et al assessed the cumulative incidence of MACE (death, acute MI, and target-vessel revascularization) and ST in a total of 2,371 patients who underwent single stenting $(\mathrm{n}=1,233)$ and multiple stenting $(\mathrm{n}=1,138)$. The 6-month cumulative incidence of MACE was $4.9 \%$ in the multiple stenting group and $3.2 \%$ in the single stenting group $(\mathrm{P}=0.04)$. A non-significant difference was also detected in favor of the single stenting group, as compared with the multiple stenting group, at the rate of ST $(0.5 \%$ vs. $0.8 \%, \mathrm{P}=0.35)$ [7]. The outcomes $(2.0 \%$ MACE, and a single incident of acute

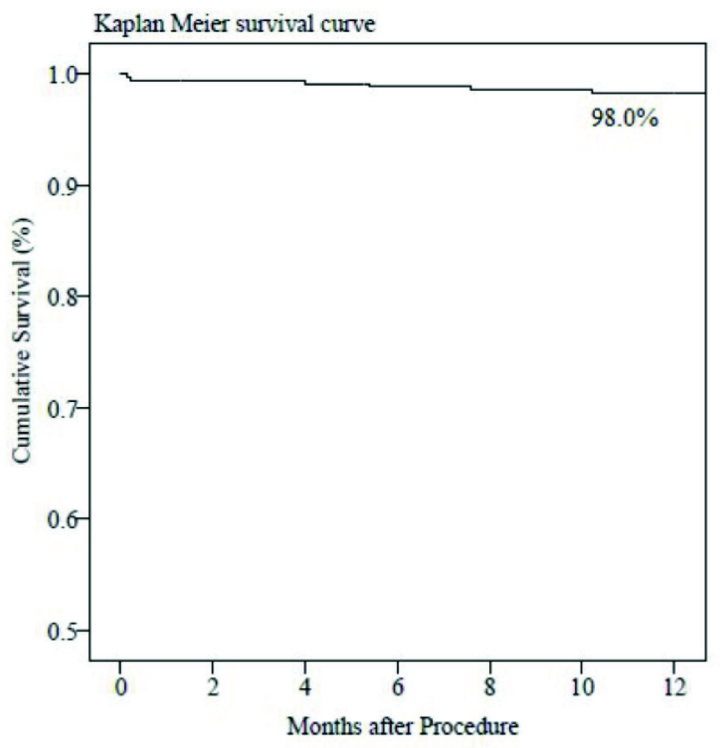

Figure 3. Time-to-event curve by Kaplan-Meier method at 12-month follow-up. 
ST) of the present study at 12-month follow-up are consistent with these previous studies.

The limitations of this study include 12-month followup period, which may not be enough for the determination of long-term safety and performance of long-tapered BioMime Morph SES system in patients with long diffused de novo coronary lesions. Moreover, this was a retrospective, nonrandomized, multicentric study and consequently, further large prospective studies with long-term follow-up are necessary. This study lacks angiographic data, and hence, we could not identify any possible complications like stent fracture at the deployment site nor the angiographic characteristics of restenosis with long-tapered coronary stent system.

\section{Conclusions}

The present study demonstrated favourable procedural success and device success in addition to optimum safety outcomes with the use of long-tapered BioMime Morph SES system in a real-world pateints with long diffused de novo coronary lesions.

\section{Acknowledgments}

The authors would like to thank Vipin Bulani, PhD, Pankaj Wagh, PhD, Nadeem Sayyed, M.Pharm and Prem Sahu, M.Sc. for their assistance in the preparation of this manuscript. Also, thanks to all the included patients in this study.

\section{Funding}

This study was partially funded by Meril Life Sciences Pvt. Ltd.

\section{Conflict of Interest}

Dr. Ashok S. Thakkar, Prakash Kumar Turiya, and Priyanka J. Desai are full-time employee of Meril Life Science, Pvt. Ltd., India. The other authors have no potential conflict of interest to declare.

\section{Disclosure}

None.

\section{References}

1. Tan CK, Tin ZL, Arshad MKM, Loh JKK, Jafary FH, Ho $\mathrm{HH}$, Ong PJL, et al. Treatment with 48-mm everolimuseluting stents : Procedural safety and 12-month patient outcome. Herz. 2018.

2. Roach MR, MacLean NF. The importance of taper proxi- mal and distal to Y-bifurcations in arteries. Front Med Biol Eng. 1993;5(2):127-133.

3. Zubaid M, Buller C, Mancini GB. Normal angiographic tapering of the coronary arteries. Can J Cardiol. 2002;18(9):973-980.

4. Sgueglia GA, Belloni F, Summaria F, Conte M, Cortese B, Silva PL, Ricci R, et al. One-year follow-up of patients treated with new-generation polymer-based $38 \mathrm{~mm}$ everolimus-eluting stent: the P38 study. Catheter Cardiovasc Interv. 2015;85(2):218-224.

5. Timmins LH, Meyer CA, Moreno MR, Moore JE, Jr. Mechanical modeling of stents deployed in tapered arteries. Ann Biomed Eng. 2008;36(12):2042-2050.

6. Ellis SG, Holmes DR. Strategic approaches in coronary intervention. 2006: Lippincott Williams \& Wilkins.

7. Bin QS, Wen LS, Bo X, Jue C, Bo LH, Jin YY, Lin CJ, et al. Clinical outcomes for single stent and multiple stents in contemporary practice. Clin Cardiol. 2009;32(9):E3339.

8. Raber L, Juni P, Loffel L, Wandel S, Cook S, Wenaweser P, Togni M, et al. Impact of stent overlap on angiographic and long-term clinical outcome in patients undergoing drug-eluting stent implantation. J Am Coll Cardiol. 2010;55(12):1178-1188.

9. Levine GN, Bates ER, Blankenship JC, Bailey SR, Bittl JA, Cercek B, Chambers CE, et al. 2011 ACCF/AHA/ SCAI Guideline for percutaneous coronary intervention: a report of the American College of Cardiology Foundation/American Heart Association Task Force on practice guidelines and the society for cardiovascular angiography and interventions. Circulation. 2011;124(23):e574-651.

10. Mendis S, Thygesen K, Kuulasmaa K, Giampaoli S, Mahonen M, Ngu Blackett K, Lisheng L, et al. World Health Organization definition of myocardial infarction: 200809 revision. Int J Epidemiol. 2011;40(1):139-146.

11. Cutlip DE, Windecker S, Mehran R, Boam A, Cohen DJ, van Es GA, Steg PG, et al. Clinical end points in coronary stent trials: a case for standardized definitions. Circulation. 2007;115(17):2344-2351.

12. Banka VS, Baker HA, 3rd, Vemuri DN, Voci G, Maniet AR. Effectiveness of decremental diameter balloon catheters (tapered balloon). Am J Cardiol. 1992;69(3):188193.

13. Kitahara H, Okada K, Kimura T, Yock PG, Lansky AJ, Popma JJ, Yeung AC, et al. Impact of stent size selection on acute and long-term outcomes after drug-eluting stent implantation in de novo coronary lesions. Circ Cardiovasc Interv. 2017;10(10):e004795.

14. Premchand RK, Kumar YS. A report of successful procedural, clinical, and angiographic outcomes with a tapered stent of a patient in naturally tapered coronary vessel. J Clin Diagn Res. 2017;11(1):OD06-OD07.

15. Sinha SK, Mahrotra A, Abhishekh NK, Razi M, Aggarwal P, Tripathi S, Rekwaal L, et al. Acute stent loss and its retrieval of a long, tapering morph stent in a tortuous, calcified lesion. Cardiol Res. 2018;9(1):63-67.

16. Valero E, Consuegra-Sanchez L, Minana G, Garcia-Blas S, Rodriguez JC, Moyano P, Sanchis J, et al. Initial experience with the novel BioMime $60 \mathrm{~mm}$-long sirolimus- 
eluting tapered stent system in long coronary lesions. EuroIntervention. 2018;13(13):1591-1594.

17. Zivelonghi C, van Kuijk JP, Nijenhuis V, Poletti E, Suttorp MJ, van der Heyden JAS, Eefting FD, et al. First report of the use of long-tapered sirolimus-eluting coronary stent for the treatment of chronic total occlusions with the hybrid algorithm. Catheter Cardiovasc Interv. 2018:1-9. 18. Matchin YG, Atanesyan RV, Kononets EN, Danilov NM, Bubnov DS, Ageev FT. [The first experience of using very long stents covered with sirolimus $(4060 \mathrm{~mm})$ in the treatment of patients with extensive and diffuse lesions of the coronary arteries]. Kardiologiia. 2017;57(4):19-26. 